\title{
Power flow in the interior and exterior of cylindrical coated nano-particles
}

\author{
Arslanagic, Samel
}

Publication date:

2012

Link back to DTU Orbit

Citation (APA):

Arslanagic, S. (2012). Power flow in the interior and exterior of cylindrical coated nano-particles. Paper presented at 3rd International Conference on Metamaterials, Photonic Crystals and Plasmonics, META'12, Paris, France.

\section{General rights}

Copyright and moral rights for the publications made accessible in the public portal are retained by the authors and/or other copyright owners and it is a condition of accessing publications that users recognise and abide by the legal requirements associated with these rights.

- Users may download and print one copy of any publication from the public portal for the purpose of private study or research.

- You may not further distribute the material or use it for any profit-making activity or commercial gain

- You may freely distribute the URL identifying the publication in the public portal

If you believe that this document breaches copyright please contact us providing details, and we will remove access to the work immediately and investigate your claim 


\title{
Power flow in the interior and exterior of cylindrical coated nano-particles
}

\author{
Samel Arslanagić \\ Department of Electrical Engineering, Electromagnetic Systems, \\ Technical University of Denmark, Denmark \\ E-mail: sar@elektro.dtu.dk
}

\begin{abstract}
Electrically small configurations consisting of resonant active coated nano-particles of a circularly cylindrical shape are considered in the case of a magnetic line source excitation at optical frequencies. The active particles are comprised of a canonical gain-impregnated silica nano-core covered concentrically with a silver, gold, or copper nanoshell. Particular attention is devoted to both the direction and the magnitude of the power flow density inside and outside of such particles. The results for the active coated nano-particles are related to those of the corresponding passive designs at optical frequencies, and are further contrasted to the results for the electrically small structures based on epsilon-negative materials at radio frequencies.
\end{abstract}

\section{Introduction}

Numerous potential applications exploiting metamaterials (MTMs), as well as plasmonic materials, have been proposed $[1,2]$, with particular attention devoted to subwavelength MTM-based [3]-[5] and plasmonic-based [6][10] particles. The gain inclusion in the active designs was found to lead to the so-called super-resonant states, which for a plane wave incidence resulted in scattering crosssections vastly surpassing values predicted by the geometrical size of the CNPs [6], [10]. Similarly, enormously large radiated powers were obtained in the case of localized excitation sources [8], [9]. These are all desirable features which make the plasmonic-based CNPs attractive candidates for nano-sensing, -lasing and amplifying applications; see e.g., a recent review [11] and the works references therein for an excellent overview of applications and potentials of plasmonic-based devices.

To better understand the interaction between the localized sources and plasmonic-based particles, and their overall radiation process, the present work investigates the power flow associated with various types of circularly cylindrical active coated nano-particles (CNPs) excited by a magnetic line source (MLS) at optical frequencies. Specifically, the active particles are comprised of a gainimpregnated silica nano-core covered concentrically with a silver, gold, or copper nano-shell where the size-dependency of the permittivity of this nano-shell is taken into account. The gain model for the active CNPs is a canonical, constant frequency, gain model. The previously reported superresonant states of the proposed active CNPs in [9], and the associated large levels of the radiated power, are correlated with the correspondingly large localizations of the power density near specific parts of the shell-interfaces and its corresponding large magnitude inside the gain region. The resulting interactions with the gain material lead to a strong and distinct dipolar power radiation in all of these electrically small, active super-resonant CNP designs. These active CNP power flow results are compared to those obtained for the corresponding passive structures at optical frequencies, and are further contrasted to epsilon-negative (ENG) sub-wavelength resonator designs at radio frequencies. The latter sheds further light on important differences between the optical and radio frequency cases. We note that interesting power flow results were reported in [5] for MTM-based cylindrical configurations for a finite radius line source excitation, as well as in [10] for active, and [11], [12] for passive nano-particles of spherical shape, against which the present findings for the cylindrical active CNPs will be displayed. Throughout the work, the time factor $\exp (j \omega t)$, with $\omega$ being the angular frequency, and $t$ being the time, is assumed and suppressed.

\section{Configuration and methods of analysis}

We consider the CNP configuration depicted in Figure 1; it consists of an infinite circular cylindrical nano-core (Region 1) covered concentrically with an infinite cylindrical nanoshell (Region 2). The inner and outer radii of the nano-shell are denoted by $\rho_{1}$ and $\rho_{2}$, respectively, and the CNP is imbedded in free-space (region 3 ) with the permittivity, $\varepsilon_{0}$, permeability, $\mu_{0}$, and wave number $k_{0}=\omega \sqrt{\varepsilon_{0} \mu_{0}}=2 \pi / \lambda$, with $\lambda$ being the free-space wavelength. The CNP is illuminated by the field provided by an arbitrarily located and infinitely long magnetic line source (MLS) possessing a constant magnetic current $I_{m}[\mathrm{~V} / \mathrm{m}]$. The nano-core and nano-shell regions of the CNP may, in general, contain an arbitrary simple, lossy and dispersive material. However, for the present purposes, specific materials are chosen for these two regions, as explained later in Section 3 of this manuscript. 
A circular cylindrical coordinate system $(\rho, \phi, z)$ and an associated rectangular coordinate system $(x, y, z)$ are introduced such that their origins coincide with the crosssectional center of the CNP, and such that the entire CNPMLS configuration is infinite in the $z$-direction, with the MLS being parallel to the axis of the cylinders. The coordinates of the observation point are $(\rho, \phi)$, while those of the MLS are $\left(\rho_{s}, \phi_{s}\right)$.

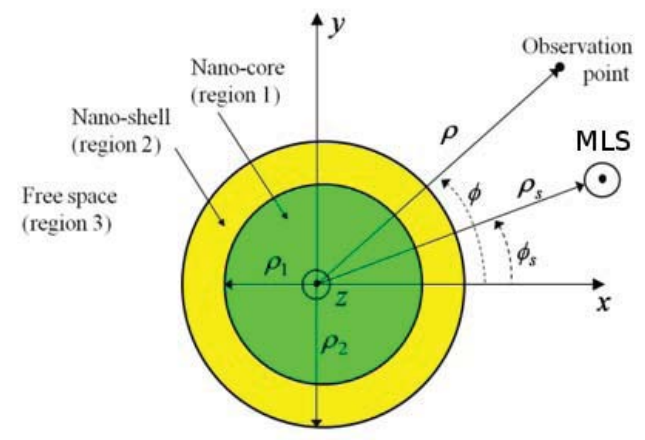

Figure 1: The MLS-excited CNP.

The analytical solution for the problem in Figure 1 has been derived in [4], and only its main features will be outlined in here. For the MLS excitation case, the magnetic field in all regions (incident and scattered fields in the region containing the MLS, and total fields in the remaining regions) contains only a $z$-component, i.e., $\boldsymbol{H}=\hat{z} H_{z}$, while the electric field in all regions has the form of $\boldsymbol{E}=\hat{\rho} E_{\rho}+\hat{\phi} E_{\phi}$, where $E_{\rho}$ and $E_{\phi}$ are its $\rho$ - and $\phi$ components. The unknown field components are expanded in cylindrical wave functions, and thus contain a number of unknown expansion coefficients. The latter follow in a straightforward manner from the application of the electromagnetic boundary conditions at the two interfaces of the CNP. These field solutions were used in [4] to perform an extensive study of electrically small passive MTM-based particles of cylindrical shape. To account for the near- and far-field properties of a number of cylindrical active plasmonic-based CNP configurations, the necessary gain levels for the so-called super-resonant state were determined recently [9]. To further address the intriguing electromagnetic properties of these particles, the field solutions from [4] are presently used to explore the flow of electromagnetic power inside and outside the CNP. This is done in terms of the Poynting vector $\boldsymbol{S}$, which is given by

$$
\begin{aligned}
\boldsymbol{S} & =(1 / 2) \operatorname{Re}\{\boldsymbol{E} \times \boldsymbol{H} *\}=\hat{x} S_{x}+\hat{y} S_{y} \\
& =\hat{x}\left[S_{\rho} \cos \phi-S_{\phi} \sin \phi\right]+\hat{y}\left\lfloor S_{\rho} \sin \phi+S_{\phi} \cos \phi\right],
\end{aligned}
$$

where $S_{x}$ and $S_{y}$ are the $x$ - and $y$-components of $\boldsymbol{S}$, while $S_{\rho}=\operatorname{Re}\left\{E_{\phi} H_{z}^{*}\right\} / 2$ and $S_{\phi}=-\operatorname{Re}\left\{E_{\rho} H_{z}^{*}\right\} / 2$ are its $\rho$ - and $\phi$-components. The asterix * designates complex conjugation. In Section 3, attention will be devoted to both the amplitude and the direction of the Poynting vector determined with (1).

\section{Results and discussions}

As reported in [6], [8]-[10], highly resonant sub-wavelength CNPs can be designed by including gain in their interiors, e.g., their nano-cores. As to the cylindrical CNPs considered in [9], the nano-core was in all cases made of silica, had a radius of $24 \mathrm{~nm}$, and was coated with a $6 \mathrm{~nm}$ thick plasmonic (silver, gold, or copper) nano-shell. The permittivity of the silica nano-core was expressed as $\varepsilon_{1}=\left(n^{2}-\kappa^{2}-2 j n \kappa\right) \varepsilon_{0}$ and was comprised of two contributions: a contribution from its refractive index in the considered frequency region $(n=\sqrt{2.05})$ and a contribution from the canonical gain model. The parameter $\kappa$ (termed the optical loss or gain constant) determines the nature of the nano-core: lossless (and passive) for $\kappa=0$, lossy and passive for $\kappa>0$ (optical loss constant) and active for $\kappa<0$ (optical gain constant). As to the plasmonic nanoshell permittivity, its empirical values, which account for its size-dependence and were used successfully in [6], were employed. In particular, for the silver-based CNP configuration excited by a MLS, the so-called superresonant state, with a large total radiated power, was identified for $\kappa=-0.175$ and the free-space excitation wavelength $\lambda=577.70 \mathrm{~nm}$ [9]. This total radiated power enhancement is shown in Figure 2 in terms of the normalized radiation resistance (NRR) as a function of the wavelength when the MLS is located at $12 \mathrm{~nm}$ along the positive $x$-axis and has the current amplitude: $I_{m}=1[\mathrm{~V} / \mathrm{m}]$.

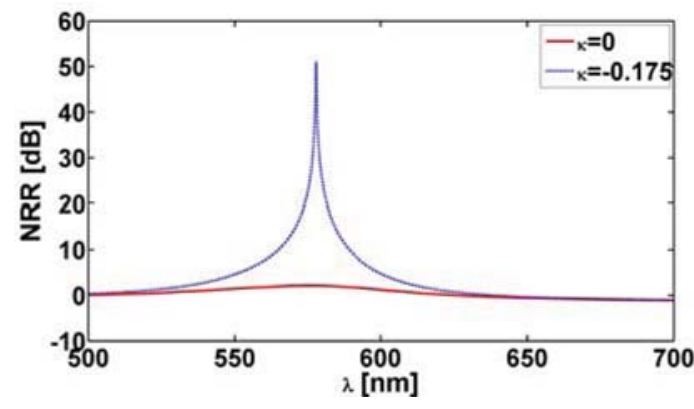

Figure 1: The NRR of a silver-based passive and superresonant active CNP.

Note that the NRR equals the total power radiated by the MLS in the presence of the CNP normalized with the power radiated by the MLS alone in free-space. As can be seen in the figure, a peak value of the $\mathrm{NRR} \approx 51 \mathrm{~dB}$ is found for the super-resonant active CNP at $577.70 \mathrm{~nm}$. This should be compared to a NRR of around $2 \mathrm{~dB}$ for the corresponding lossless and passive CNP $(\kappa=0)$, which also is included in the figure. As explained in [9] in terms of the near-field distributions of the magnetic field, the super-resonance behavior reported in Figure 2 for the active silver-based CNP is due to a strong excitation of the resonant dipole 
mode inside the CNP. The existence of the resonant dipole mode inside an electrically small CNP (diameter size of approximately $\lambda / 10$ ) is a result of juxtaposing of two materials, one with positive permittivity (silica nano-core) and the other with a negative permittivity (silver nano-shell) [1]. The strong excitation of this dipole mode is heavily assisted by the gain-impregnation of the silica-nano core; its presence overcomes the plasmonic material losses [6].

The super-resonant state of the active Ag-based CNP is next illustrated in Figure 3a). The magnitude (color) and direction (arrows) of the Poynting vector (1) is shown when the MLS is located at $12 \mathrm{~nm}$ along the positive $x$-axis.

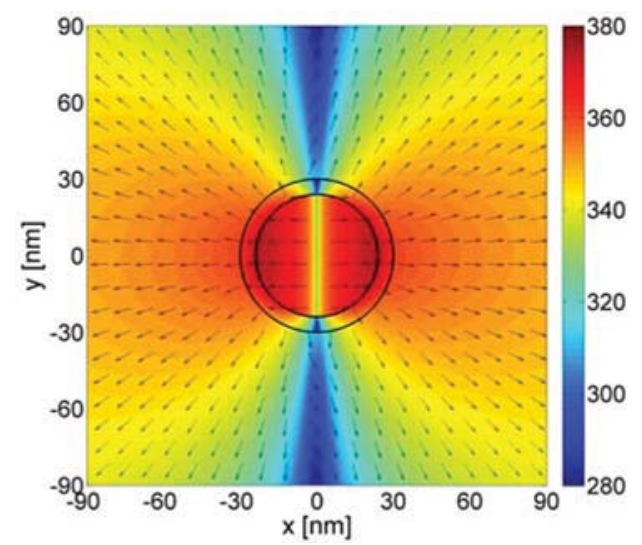

(a)

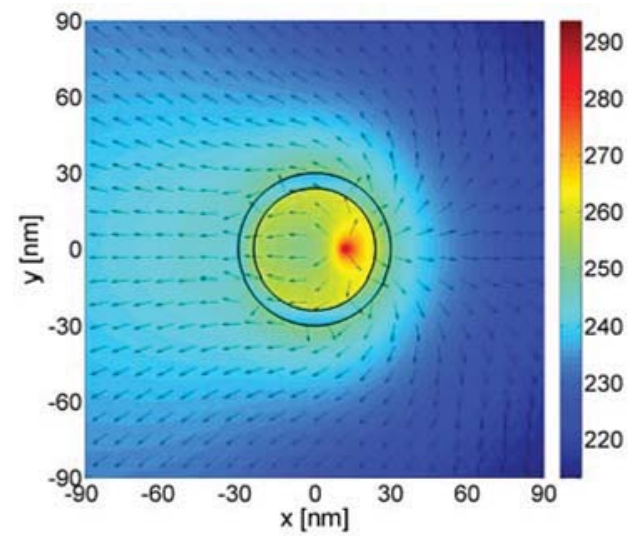

(b)

Figure 3: The magnitude (color) and direction (arrows) of the power flow density for the super-resonant active Agbased CNP (a) and for the corresponding passive Ag-based CNP (b). In both cases, the radius of the silica nano-core is $24 \mathrm{~nm}$, the thickness of the plasmonic nano-shell is $6 \mathrm{~nm}$, and the MLS is located at $12 \mathrm{~nm}$ along the positive $x$-axis. Note that the dynamic range in (a) is larger than that in (b). The curves representing the cylindrical surfaces of the CNP are included in the figure.

A very clear and strong dipolar pattern, as though it is being produced by a dipolar source of radiation centered at the origin of the CNP, is observed in this figure. The power density is rather strong and homogeneous in strength and direction inside the CNP. It is mainly directed along the negative and positive $x$-direction in the left and right halves, respectively, of the nano-core. This is particularly true near the inner surface of the nano-shell, where one witnesses a profound localization of the power density within the CNP. The interaction of the large fields in the electrically small resonant CNP with the canonical gain inside the nano-core leads to a clear and strong dipolar radiated power pattern. This radiated power behavior is illustrated with by an outward-propagating Poynting vector from the surface of the CNP in Figure 3a). While the magnitude of the Poynting vector suggests the pattern is symmetric, its direction reveals a slight asymmetry which is attributed to the off-set MLS location in this case. In contrast to the super-resonant Agbased CNP results, Figure $3 \mathrm{~b}$ ) shows the magnitude and the direction of the corresponding passive Ag-based CNP. No excitation of a resonant dipole mode is in evidence; this is consistent with the very low radiated power levels observed, e.g., in Figure 2.

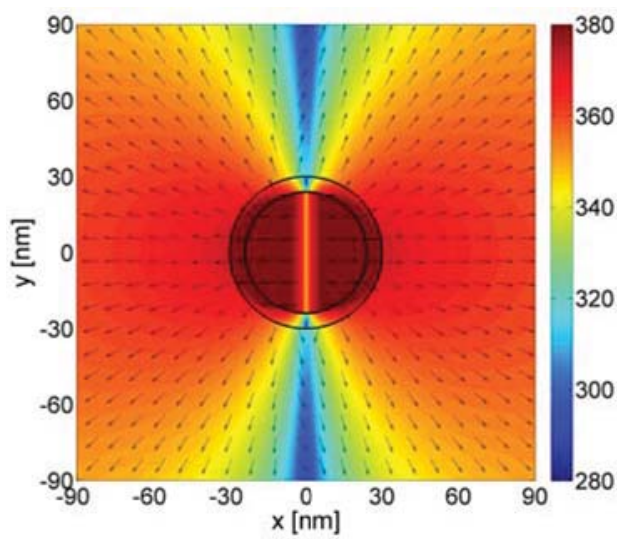

(a)

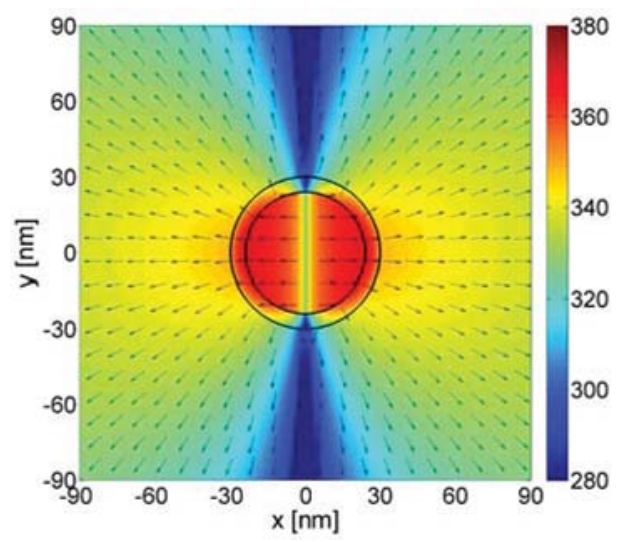

(b)

Figure 4: The magnitude (color) and direction (arrows) of the power flow density for the super-resonant active Aubased CNP (a) and Cu-based CNP (b). In both cases, the radius of the silica nano-core is $24 \mathrm{~nm}$, the thickness of the plasmonic nano-shell is $6 \mathrm{~nm}$, and the MLS is located at 12 $\mathrm{nm}$ along the positive $x$-axis. The curves representing the cylindrical surfaces of the CNP are included in the figure.

Besides the Ag-based CNP configuration considered above, 
the corresponding super-resonant CNPs with their nanoshell composed of either gold or copper have also been studied [9]. For the Au-based CNP, the super-resonance was identified for $\kappa=-0.262$ and $\lambda=669.39 \mathrm{~nm}$, with a resulting $\mathrm{NRR} \approx 56 \mathrm{~dB}$. For the $\mathrm{Cu}$-based $\mathrm{CNP}$, it was identified for $\kappa=-0.310$ and $\lambda=662.19 \mathrm{~nm}$, with a resulting $\mathrm{NRR} \approx 46 \mathrm{~dB}$. The behavior of the latter two CNPs with the MLS located at $12 \mathrm{~nm}$ along the positive $x$-axis is illustrated further with the power flow density results reported for the Au-based CNP in Figure 4a) and for the $\mathrm{Cu}$ based CNP in Figure 4b). Again, the power flow clearly has a dipolar form. However, its magnitude for the Au-based CNP surpasses the values for the other two CNPs given in Figures 3a) and 4b). Because it has the highest losses, the corresponding value for the super-resonant $\mathrm{Cu}$-based CNP is the lowest of all of the super-resonant cases. The large levels of the power flow density for the Au-based CNP correspond to a strong localization of the power density within the CNP, which in turn leads to the largest NRR values of the three super-resonant CNP cases. The relatively low levels for the $\mathrm{Cu}$-based CNP are perfectly in line with the correspondingly lowest NRR values.

We note that for all super-resonant CNPs cases considered above, the power flow has also been observed in an extended range up to $200 \mathrm{~nm}$ in both the $x$ - and $y$-directions. Throughout such a range, a clear dipolar pattern, suggesting an outwardly propagating power flow density away from the CNP, was observed. Moreover, power flow results were obtained for super-resonant CNPs $(\kappa=-0.175)$ for wavelengths shorter $(\lambda=567.70)$ and longer $(\lambda=587.70)$ than the super-resonant state wavelength $(\lambda=577.70)$. Although they are not included here, these results still show dipolar patterns with an outwardly propagating power flow density similar to those found in Figures 3a) and 4, but with a lower strength. Thus, contrary to power flows for active [10] and passive [13] nano-particles of spherical shape, which were found to exhibit specific vortices near their plasmon resonances, this is not the case for the (infinite and, hence, effectively two-dimensional) active super-resonant CNPs of cylindrical shape investigated here.

The above results for the electrically small super-resonant cylindrical CNPs, which employ the naturally occurring ENG-based metals at optical frequencies, are next contrasted to the analogous electrically small structures at radio frequencies which must utilize ENG metamaterials. To this end, Figure 5a) shows the NRR as a function of frequency, $f$, for a resonant sub-wavelength ENG shell with an inner radius of $6 \mathrm{~mm}$ and an outer radius of $10.03 \mathrm{~mm}$. The ENG shell is modeled by a lossless Drude dispersion model [1] adjusted to select the relative permittivity of the shell to be 3.0 at the design frequency of $300 \mathrm{MHz}$ (giving the diameter of the particle to be $\lambda / 50)$. The so-selected geometrical and electrical parameters of the shell are in compliance with the well-known resonance conditions in electrically small structures of cylindrical shape [3], [4]. Figure 5a), which was obtained when the MLS was located along the $x$-axis at $3 \mathrm{~mm}$, shows a resonance phenomenon with the NRR $\approx 17$
$\mathrm{dB}$ at $300 \mathrm{MHz}$. The associated power flow density (magnitude and direction) is depicted in Figure 5b). It is found to differ significantly from any of the super-resonant active CNP cases. In this case, the power flow lines are seen to exhibit an interesting behavior, including vortices, at the three "deep-blue" spots - one to the left of the particle and two inside the shell-region. In fact, the power flow outside of the resonant ENG-based particle resembles the one for the lossless and passive CNP in Figure 3b), rather than that of the super-resonant active CNP. In the latter, the significantly smaller physical size of the particle, as well as the gain inclusion, are the driving factors behind the clear and strong outward power flow and the dipolar shape of the response.

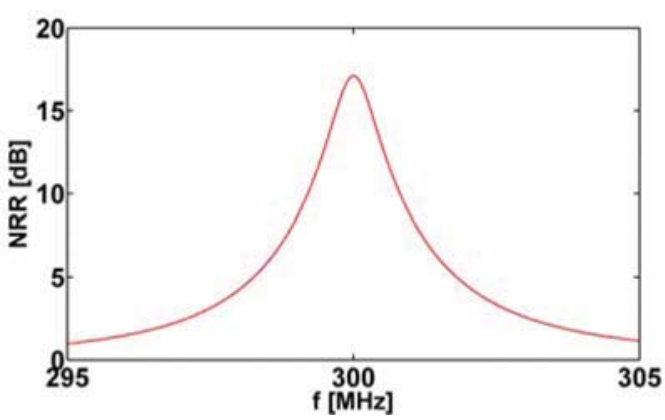

(a)

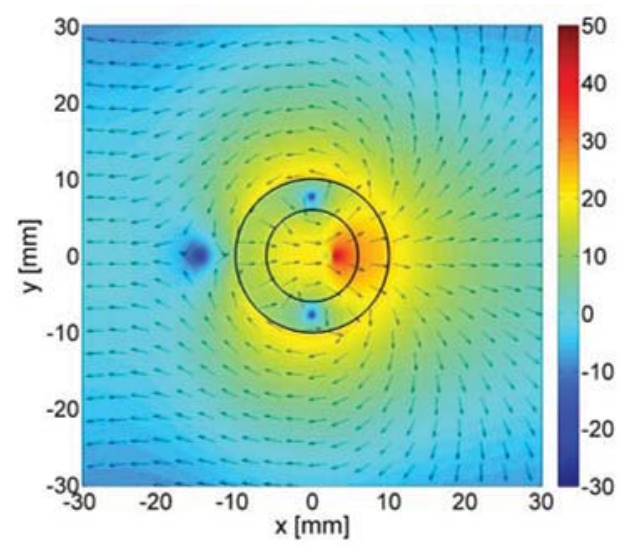

(b)

Figure 5: (a) The NRR as a function of frequency, $f$, for an ENG shell. (b) The magnitude (color) and direction (arrows) of the power flow density for the resonant ENG-shell at 300 MHz. In (a) and (b), the MLS is at $3 \mathrm{~mm}$ along the positive $x$-axis. Note that the curves representing the cylindrical surfaces of the ENG shell are also included in (b).

\section{Summary and conclusions}

The power flow density (magnitude and direction) in the interior and exterior of electrically small configurations consisting of resonant active coated nano-particles (CNPs) of a circularly cylindrical shape was examined. The source of excitation was taken to be an infinitely long magnetic line source (MLS) at optical frequencies. The active particles were comprised of a canonical gain-impregnated silica nano- 
core covered concentrically with a silver, gold, or copper nano-shell. The previously reported super-resonant states, excited in silver-, gold-, and copper-based CNPs and leading to large powers extracted from the MLS for a given value of the current along it, were confirmed with the power flow density results. For all cases, the power density was found to be rather strong and homogeneous in strength and direction inside the CNP; this being particularly so near the inner surface of the nano-shell, where a profound localization of the power density within the CNP was observed. The interaction of such large fields with the canonical gain inside the nano-core was found to lead to a clear and strong dipolar power pattern, with an outwardly propagating power flow density. These effects were found to be most notable for the gold-based CNP, as it is the least lossy case of all at the resonance wavelength. Moreover, no peculiar behavior in terms of certain vortices was found for the cylindrical active and super-resonant active CNPs at specific wavelengths that are shorter or longer than the resonant wavelength, this appearing in contrast to the observations for spherical particles [10], [13]. The results for the active CNPs at optical frequencies were furthermore contrasted to those obtained at radio frequencies for the corresponding epsilonnegative based particles. The latter resulted in power flow behavior significantly different from any of the superresonant active optical CNP cases. In fact, the power flow lines in the radio frequency case were found to exhibit an interesting behavior including vortices at specific locations in the interior and exterior of the particle.

\section{References}

[1] N. Engheta, and R. W. Ziolkowski, MetamaterialsPhysics and Engineering Applications, John Wiley \& Sons, New York, 2006.

[2] W. Cai, and V. Shalaev, Optical Metamaterials, Springer, Berlin, 2010.

[3] A. Alú, and N. Engheta, "Polarizabilities and effective parameters for collections of spherical nano-particles of concentric double-negative (DNG), single-negative (SNG) and/or double-positive (DPS) metamaterial layers," J. Appl. Phys., vol. 97, 094 310, May 2005.

[4] S. Arslanagić, R. W. Ziolkowski, and O. Breinbjerg, "Analytical and numerical investigation of the radiation and scattering from concentric metamaterial cylinders excited by an electric line source," Radio Sci., vol. 42, RS6S15, doi: 10.1029/2007RS 003644, Nov. 2007.

[5] S. Arslanagić, N. C. J. Clausen, R. R. Pedersen, and O. Breinbjerg, "Properties of sub-wavlength resonances in metamaterial cylinders," NATO Advanced Research Workshop - Metamaterials for Secure Information and Communication Technologies, Marokko, 2008.

[6] J. A. Gordon, and R. W. Ziolkowski, "The design and simulated performance of a coated nano-particle laser," Opt. Exp., vol. 15, issue 5, pp. 2622-2653, March 2007.

[7] A. Alú, and N. Engheta, "Plasmonic and metamaterial cloaking: physical mechanisms and potentials," J. Opt.
A: Pure Appl. Opt., 10, 2008, doi: 10.1088/14644258/10/9/093002.

[8] S. Arslanagić, and R. W. Ziolkowski, "Active coated nano-particle excited by an arbitrarily located electric Hertzian dipole - resonance and transparency effects," J. Opt., Vol. 12, 024014, 2010.

[9] S. Arslanagić, Liu, Y., Malureanu, R., and R. W. Ziolkowski, "Impact of the excitation source and plasmonic material on cylindrical active coated nanoparticles," Sensors, Vol. 11, 9109-9120, 2011.

[10] S. D. Campbell, and R. W. Ziolkowski, "Impact of strong localization of the incident power flow density on the nano-amplifier characteristics of active coated nano-particles," Opt. Commun., In Press, Available online 19 November 2011.

[11] N. J. Halas, "Plasmonics: an emerging field fostered by Nano Letters," Nano Lett., 10, pp. 3816-3822, doi: 10.1021/n11032342, 2010.

[12]Z. B. Wang, B. S. Luk'yanchuk, M. H. Hong, Y. Lin, and T.C. Chong, "Energy flow around a small particle investigated by classical Mie theory," Phys. Rev. B, 70,035418, 2004.

[13] M. V. Bashevoy, V. A. Fedotov, and N. I. Zheludev, "Optical whirlpool on an absorbing metallic nanoparticle," Opt. Exp., vol. 13, no. 21, pp. 8372-8379. 